\title{
Conversas sobre serviços públicos
}

\author{
Bruna Diirr $^{1,2^{*}}$, Renata Mendes de Araujo ${ }^{1,2 \dagger}$, Claudia Cappelli ${ }^{1,2}$ \\ ${ }^{1}$ Programa de Pós-Graduação em Informática - Universidade Federal do Estado do Rio \\ de Janeiro (UNIRIO) \\ Rio de Janeiro - RJ - Brasil \\ ${ }^{2}$ Núcleo de Pesquisa e Prática em Tecnologia - UNIRIO \\ Rio de Janeiro - RJ - Brasil \\ \{bruna.diirr, renata.araujo, claudia.cappelli\}@uniriotec.br
}

\begin{abstract}
Many discussions enforce the need to encourage involvement and participation of Society in public issues. It is argued that the use of conversations on public services encourages closer ties between Society and Government. This paper presents a tool to support discussion and sharing of information about the provision process of public service. It enables that the information obtained can be used to understand the discussion and for identification of improvements in services.
\end{abstract}

Resumo. Várias discussões reforçam a necessidade de estimular o envolvimento e a participação da Sociedade nos assuntos públicos. Argumenta-se que o estímulo e a facilitação das conversas sobre os serviços públicos nos quais os cidadãos estejam envolvidos aumente a aproximação entre Sociedade e Governo. Este artigo apresenta uma ferramenta de apoio à discussão e compartilhamento de informações sobre os processos de prestação de serviços públicos, possibilitando que as informações obtidas sejam utilizadas no entendimento da discussão e como fonte de análise para a identificação de melhorias nos serviços prestados.

\section{Introdução}

“- O senhor teve um longo prazo a seu dispor para fazer quaisquer sugestões ou reclamações, como o senhor sabe-disse o Sr. Prosser.

- Um longo prazo? - exclamou Arthur. - Longo prazo? Eu só soube dessa história quando chegou um operário na minha casa ontem. Perguntei a ele se tinha vindo para lavar as janelas e ele respondeu que não, vinha para demolir a casa. É claro que não me disse isso logo. Claro que não. Primeiro lavou umas duas janelas e me cobrou cinco pratas. Depois é que me contou.

- Mas, Sr. Dent, o projeto estava à sua disposição na Secretaria de Obras há nove meses.

\footnotetext{
* Bolsita CAPES-DS

${ }^{\dagger}$ Bolsista produtividade em pesquisa $\mathrm{CNPq}$
} 
- Pois é. Assim que eu soube fui lá me informar, ontem à tarde. Vocês não se esforçaram muito para divulgar o projeto, não é verdade? Quer dizer, não chegaram a comunicar às pessoas nem nada.

- Mas o projeto estava em exposição...

- Em exposição? Tive que descer ao porão pra encontrar o projeto.

- É no porão que os projetos ficam em exposição.

- Com uma lanterna.

- Ah, provavelmente estava faltando luz.

- Faltavam as escadas, também.

- Mas, afinal, o senhor encontrou o projeto, não foi?

- Encontrei, sim - disse Arthur. - Estava em exibição no fundo de um arquivo trancado, jogado num banheiro fora de uso, cuja porta tinha a placa: Cuidado com o leopardo." [ADAMNS, 1979]

Apesar de escrito no final da década de 70, o trecho da ficção acima descreve uma situação comumente encontrada nos órgãos governamentais: distanciamento entre Governo e Sociedade no que tange às informações sobre os processos de prestação de serviços públicos. Muitas informações de interesse público estão indisponíveis para consulta da Sociedade ou, quando disponíveis, encontram-se desorganizadas, têm difícil acesso e têm nenhuma ou pouca explicação [SILVA, 2005].

Algumas iniciativas governamentais tentam disponibilizar informações aos cidadãos sobre os processos de prestação dos serviços públicos, visando estimular um maior envolvimento da Sociedade nesses assuntos [MAIA, 2001][MACINTOSH, 2004][SILVA, 2005]. Podemos citar a Carta de Serviços ao Cidadão [BRASIL, 2008], documento que deve ser elaborado por toda instituição pública de modo a informar aos cidadãos quais os serviços prestados, como acessar e obter esses serviços e quais são os compromissos de atendimento estabelecidos por esta organização pública. Outra iniciativa são as consultas públicas, divulgadas por diferentes órgãos do governo, para colher contribuições de setores especializados e da Sociedade em geral, sobre ações a serem realizadas em diferentes temas públicos [MINISTERIO DA CULTURA, 2009][MINISTÉRIO DA JUSTIÇA, 2010][MINISTÉRIO DA SAÚDE, 2010].

Pesquisas nas áreas de Democracia Eletrônica (e-democracia) e Governo Eletrônico (e-Gov) reforçam o potencial das Tecnologias de Informação e Comunicação (TICs) no surgimento de novas possibilidades de acesso à informação pelos cidadãos. As TICs oferecem a oportunidade de abertura do Governo aos Cidadãos, expondo seus trabalhos internos, informações e serviços prestados; reconhece novos canais e formas de divulgação de informações aos cidadãos; e fazem melhor uso das ferramentas para aumentar a consciência e participação dos cidadãos [ALLEN, 2004][BRYANT, 2006][HAGUE, 1999][OATES, 2008][SHIRKY, 2008][W3C, 2009].

Nas discussões sobre esses temas, o envolvimento da Sociedade segue uma escala crescente de participação [GOMES, 2004]. Argumenta-se que a aproximação entre Governo e Sociedade inicia-se a partir dos níveis mais básicos de participação, principalmente através da disponibilização de informações sobre os serviços públicos 
prestados. Contudo, pesquisas indicam que, no Brasil, ainda existem dificuldades em encontrar soluções eletrônicas efetivas que forneçam informações e possibilidades de participação da Sociedade nos assuntos públicos [SILVA, 2005][DUTTA; MIA, 2010], implicando em um distanciamento entre Sociedade e Governo.

O objetivo desse trabalho é sugerir ferramental que estimule e facilite a conversas sobre os serviços públicos prestados entre Sociedade e Governo. Para tal, propõe-se que os serviços oferecidos sejam apresentados sob a forma de processos, possibilitando um maior entendimento sobre o funcionamento do serviço. A partir dos processos apresentados, os participantes (Sociedade e Governo) podem manifestar-se de maneira global ou situada. As manifestações são organizadas de forma que possibilitem a percepção das mesmas para novos participantes e que sirvam como base para a identificação de melhorias nos serviços prestados.

O artigo está estruturado da seguinte forma: a seção 2 apresenta como a Sociedade vem interagindo como Governo através do uso de TICs no que tange os assuntos públicos; na seção 3 é descrita a proposta de conversas sobre processos públicos; a seção 4 descreve o estudo exploratório, realizado na Escola de Informática Aplicada (EIA) da UNIRIO, com objetivo de avaliar a proposta; a seção 5 apresenta um ambiente de apoio às conversas sobre os serviços públicos prestados; e, por fim, na seção 6, são expostas as conclusões obtidas com o desenvolvimento deste trabalho.

\section{Interação entre Sociedade e Governo nos assuntos públicos}

Muito se discute sobre o estímulo ao maior envolvimento da Sociedade nos assuntos públicos. Na literatura encontram-se algumas classificações de diferentes níveis de participação em contextos democráticos através da utilização de Tecnologias de Informação e Comunicação [ARNSTEIN, 1969][FEMERS; WIEDEMANN, 1993][OECD, 2001][GOMES, 2004]. Essas classificações apresentam os graus de participação democrática em uma escala crescente, que vai desde a oferta de informações e serviços pelo Governo até a deliberação de assuntos por parte da Sociedade. A cada grau identificado, incrementa-se o poder de participação, discussão e tomada de decisão da Sociedade no processo decisório.

As barreiras à participação da Sociedade em cada um dos níveis de participação envolvem aspectos tecnológicos, sociais, culturais e econômicos, como falta de acesso à infraestrutura básica, preocupação com proteção e segurança dos dados, dificuldade em encontrar informações, dificuldade de utilizar as informações e serviços disponíveis, ineficiência do retorno às solicitações, falta de conhecimento da existência das informações, preferência pelo atendimento presencial, falta de habilidade com o computador, entre outros [CGI.BR, 2010][DUTTA; MIA, 2010].

Além disso, pesquisas na área de Democracia Eletrônica indicam que, no Brasil, ainda existe grande dificuldade em encontrar soluções efetivas que estimulem a participação da Sociedade nos assuntos públicos. O relatório do Fórum Econômico Mundial sobre o uso de TICs pelos Governos aponta o Brasil como o $41^{\circ}$ no ranking relacionado a e-participação, que avalia a qualidade, relevância, utilidade e disponibilidade dos sites do Governo para o fornecimento de informações e serviços online, além de ferramentas participativas para os Cidadãos [DUTTA; MIA, 2010]. As soluções, quando disponíveis, limitam-se ao acesso às informações e serviços para 
consulta dos cidadãos, encontram-se muitas vezes desorganizadas, de difícil acesso e com nenhuma ou pouca explicação sobre o que foi disponibilizado [SILVA, 2005]. Isso implica em um distanciamento entre quem é responsável pela definição e monitoramento dos assuntos públicos (Governo) e quem é diretamente afetado pela aplicação dos mesmos (Sociedade). Percebe-se, portanto que ainda existem diversos problemas nos níveis mais básicos de participação [W3C, 2009].

Dessa forma, o foco do presente trabalho está na prestação de serviços públicos pelo Governo e no uso destes serviços pela Sociedade. Essa interação compreende as seguintes características: (i) a disponibilidade de informações relevantes sobre os serviços públicos; (ii) a possibilidade de manifestação pelos envolvidos sobre os serviços públicos prestados; (iii) a utilização das manifestações como forma de discussão e articulação cidadã sobre os serviços públicos prestados e (iv) a organização das informações geradas durante as manifestações como insumo para a identificação de melhorias na prestação de serviços.

A pesquisa da CGI.br [CGI.BR, 2010] reforça o potencial dos serviços como forma de aproximação entre Sociedade e Governo, uma vez que indica que $79 \%$ das organizações usam serviços eletrônicos oferecidos pelo Governo, $56 \%$ da população escolheria a Internet para usar os serviços na próxima vez que necessitasse e outros $60 \%$ declaram-se propensos a indicar o serviço na web para suas redes de contato.

Usualmente, a forma que a Sociedade tem para se manifestar sobre determinado serviço se dá através dos "Fale Conosco" de sites e das Ouvidorias, disponibilizados pelos órgãos públicos responsáveis pela prestação do mesmo. É possível enviar mensagens que são pré-classificadas pelo Cidadão (sugestão, elogio, crítica etc), recebidas por algum agente interno do órgão público responsável e respondidas ou não, de acordo com a política de relacionamento do órgão em questão. Exemplos desse tipo de interação são a Ouvidoria do site da Prefeitura do Rio de Janeiro [PORTAL DA PREFEITURA DO RIO, 2010], onde há possibilidade de esclarecimento de dúvidas, cadastramento de alguma solicitação e acompanhamento de uma solicitação feita, e o "Fale com a Presidenta" e "Participe do portal" do site da Presidência do Brasil [BRASIL, 2010], onde é possível enviar mensagens para o gabinete presidencial e enviar críticas e sugestões sobre o conteúdo disponibilizado no portal.

Recorrendo à literatura, também são encontrados trabalhos que tratam a aproximação entre Governo e Sociedade no que tange os serviços prestados. Hwang e Manandhar (2009) e Miah et al (2009) propõem ambientes onde são disponibilizados uma série de serviços públicos para a Sociedade. Os cidadãos podem requisitar a utilização desses serviços e acompanhar suas solicitações sem precisar comparecer a um órgão governamental.

Outra proposta é descrita por Reuver et al (2010). É apresentada uma plataforma de interface móvel baseada em wiki, que permite que os cidadãos obtenham e contribuam com novas informações que indiquem problemas desconhecidos pelos responsáveis pela informação (ex.: Uma passagem bloqueada é identificada por um cidadão que indica a localização do bloqueio na plataforma. Essa indicação será direcionada para o departamento responsável pela solução do problema). 
Apesar do potencial de uso de serviços como forma de aproximação entre Sociedade e Governo, grande parte das soluções limita-se à oferta dos serviços em um ambiente online, de modo a diminuir a burocracia e fornecer uma maior agilidade no processo. As soluções que propõem manter um maior diálogo com a Sociedade, limitam-se à possibilidade de votação em assuntos pré-estabelecidos e consulta de informações que podem ou não ser usadas pelo Governo para a melhoria do serviço. Além disso, a interação permanece somente entre Governo e Sociedade, não existindo possibilidades para uma maior interação e discussão entre os próprios cidadãos.

\section{Conversas sobre processos de prestação de serviços públicos}

Para que uma maior aproximação entre Sociedade e Governo ocorra, é proposta uma solução baseada em conversas sobre os serviços públicos prestados. Conversas são relatos feitos pelos envolvidos durante a discussão sobre determinado serviço, que visam explicar, exemplificar e corrigir as informações existentes sobre o mesmo. Elas ajudam no fornecimento de informações sobre o serviço discutido, no aumento do entendimento dos participantes sobre esse serviço, na articulação de ações pelos participantes da discussão e na explicitação de informações relevantes para a melhoria do serviço prestado.

A primeira característica dessa solução para aproximação entre Sociedade e Governo está relacionada à disponibilização dos serviços públicos que servirão como base para as conversas dos envolvidos com o mesmo. Essa disponibilização é necessária para que os envolvidos conheçam o funcionamento do serviço prestado e possam ter um maior entendimento sobre o mesmo. Para tal, propõe-se que os serviços oferecidos sejam apresentados sob a forma de modelos de processos [BUSINESS DICTIONARY, 2010].

Existem diferentes formas de apresentação de processos, que vão desde representações textuais até representações gráficas, como fluxogramas, IDEF, SIPOC, BPMN, UML, EPC, i*, Redes Petri etc. [GONÇALVES et al, 2005][CARVALHO, 2009]. Este trabalho argumenta que, independente da representação de processos adotada, é possível abstraí-la desde que sejam apresentados os elementos principais que compõem um processo: objetivo (o porquê da existência do processo), executores (quem realiza cada atividade do processo), atividades (passos do processo), artefatos (entradas e saídas das atividades), recursos (equipamentos ou sistemas de apoio às atividades), eventos (elementos disparadores do processo), regras de negócio (leis que regem o processo) e caminhos possíveis (decisões e alternativas no processo). Esses elementos auxiliam a explicação do funcionamento do serviço disponibilizado.

A segunda característica desta solução está relacionada à possibilidade de manifestações por parte dos envolvidos na conversa sobre o serviço. A solução deve permitir a manifestação dos participantes, além de orientar que a conversa mantenha-se focada no processo de prestação do serviço. Sendo assim, propõe-se que os elementos de processos sirvam como focalizadores das manifestações feitas pelos envolvidos e existam associações entre os elementos deste processo e as manifestações feitas.

A terceira característica está relacionada à existência de mecanismos que auxiliem na percepção dos participantes sobre as manifestações feitas. Ou seja, mecanismos que permitam a percepção do andamento da conversa. Esses mecanismos 
são úteis para os cidadãos por despertarem o interesse em descobrir o que está sendo discutido em determinado ponto do processo e auxiliarem no acompanhamento do que foi tratado enquanto não se estava participando da conversa; e para o Governo, pois evidenciam a existência de manifestações em determinados pontos do processo, auxiliando assim a descoberta, captura e organização das informações que podem ser relevantes para a melhoria do serviço.

A última característica da solução está relacionada à classificação das manifestações feitas durante a conversa sobre o serviço público. As conversas sobre um serviço público tendem a gerar um grande número de manifestações. A classificação das manifestações auxilia na organização das mesmas, facilitando o reconhecimento dos tipos de relatos feitos pelos participantes sobre o processo em pauta e a identificação de informações que podem ser relevantes para a melhoria do serviço.

\section{Estudo exploratório}

Um estudo exploratório foi realizado no contexto da Escola de Informática Aplicada (EIA) da UNIRIO. A EIA, por fazer parte de uma instituição de ensino pública, é um ambiente que apresenta as características e necessidades dos ambientes democráticos, como representatividade, discussão deliberativa, participação, tomada de decisão, entre outras.

Apesar de ser um contexto mais restrito do que outros órgãos públicos, os integrantes da EIA nem sempre tem conhecimento sobre o funcionamento dos serviços prestados. Assim, o serviço de inscrição de veteranos nas disciplinas oferecidas pela EIA foi modelado usando-se uma combinação de fluxos de trabalho e descrição textual e ficou disponível para os envolvidos no endereço www.uniriotec.br/mediawiki/ wikiprocess, no período de 13/10/2010 a 15/11/2010. Toda a comunidade da EIA (docentes, discentes e técnicos administrativos) foi convidada a participar do estudo e 7 integrantes, sendo 1 docente e 6 discentes (entre matriculados e egressos), aceitaram o convite. Solicitou-se a esses participantes que navegassem pelo ambiente para conhecerem os processos disponibilizados, além de comentarem e discutirem entre si os pontos que julgassem necessários. Foram identificadas 12 manifestações dos participantes, sendo 7 manifestações relacionadas ao processo "Inscrever veterano em disciplina" como um todo e 5 manifestações relacionadas a alguma atividade desse mesmo processo.

O estudo exploratório teve como objetivo (a) explorar a receptividade da proposta de utilização de conversas por parte dos envolvidos com os serviços prestados pela EIA; (b) avaliar a forma de associação entre as manifestações dos participantes e os elementos de modelo de processos; (c) identificar os principais tipos de manifestações e (d) identificar os problemas e as dificuldades enfrentadas pelos participantes durante a interação.

Os resultados do estudo indicaram que há um potencial para promover conversas sobre os serviços fornecidos por uma organização tendo-se como base os processos que descrevem o seu funcionamento. Ao longo do estudo, as informações previamente fornecidas sobre o processo (descrição textual e representação gráfica) despertaram interesse nos participantes, que utilizaram essas informações para manifestarem-se sobre o processo disponibilizado. As manifestações apresentaram novas informações sobre o 
processo, problemas nas informações fornecidas previamente e sugestões para a solução das dificuldades e inconsitências relatadas. Em todas as manifestações feitas, os participantes referenciaram e discutiram os elementos que compõem o processo, mesmo sem conhecimento teórico ou prático em processos (Figura 1). A referência implícita aos elementos fez com que a conversa se mantivesse focada no processo em pauta, isto é, na "Inscrição de veteranos em disciplinas", não existindo comentários cujo assunto fosse outro que não este processo.

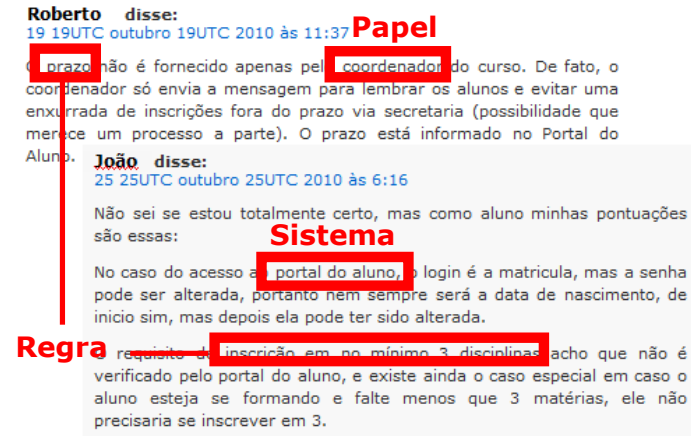

Figura 1. Referência aos elementos de processos

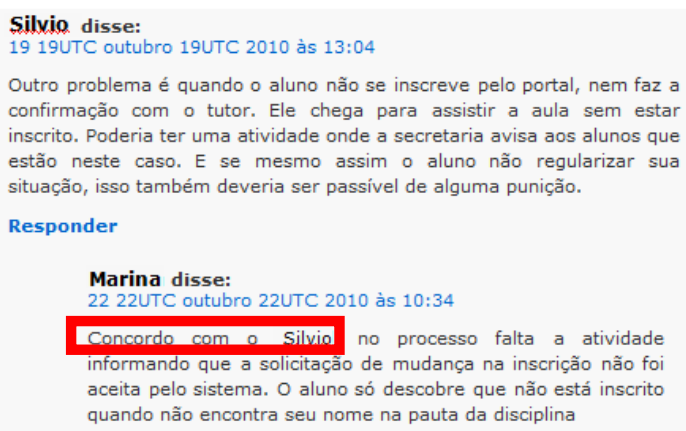

Figura 2. Referência a manifestações de outros participantes

Além disso, notou-se também referências explícitas sobre as manifestações de outros participantes (Figura 2), configurando conversas. Essas referências foram utilizadas na argumentação de uma nova manifestação ou para complementar ou corrigir as informações fornecidas anteriormente. Isso promoveu o estabelecimento de um contato entre os participantes, o que ajudou no aumento do conhecimento sobre o processo em discussão além de promover discussões e sugestões de possíveis melhorias ao processo apresentado.

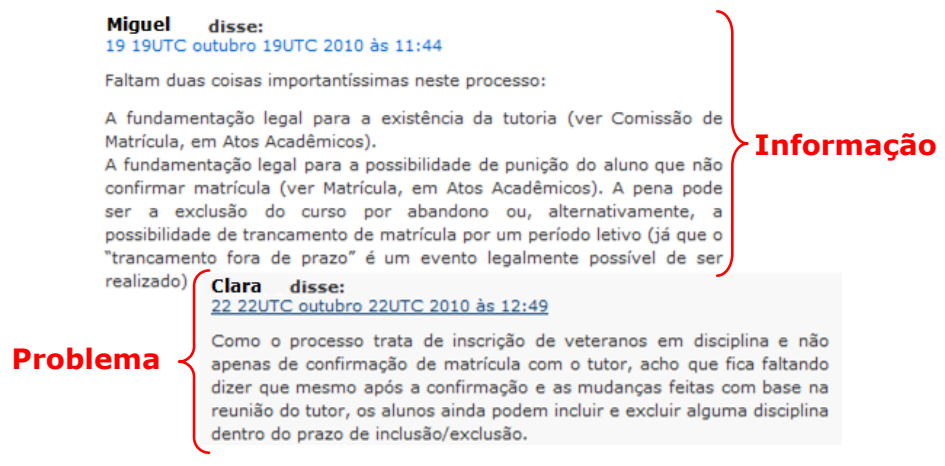

Figura 3. Tipos de manifestações sobre o serviço

O estudo exploratório serviu também para a identificação de tipos de manifestações possíveis durante a discussão de serviços prestados por uma organização. Ao longo do estudo foi possível identificar relatos de experiências (discentes só descobrem que não estavam inscritos quando não encontraram seu nome na pauta da disciplina), problemas (a regra que impõe um número mínimo de disciplinas inscritas pode não ser verificada pelo sistema de inscrição), sugestões (a confirmação de inscrição junto ao tutor só seria feita no caso dos alunos que tivessem alguma pendência ou quisessem alterar algo no pedido de inscrição), informações (além de divulgado pelo coodenador, o prazo para inscrição em disciplinas também é informado no sistema de 
inscrição) (Figura 3). Os tipos de manifestações identificados servirão como base para a classificação e organização das manifestações feitas na solução de apoio à aproximação entre Sociedade e Governo (quarta característica de interação entre Governo e Sociedade no que tange a prestação de serviços públicos).

\section{Ambiente de apoio às conversas sobre os serviços públicos prestados}

Baseado na análise do estudo exploratório, foi especificado um ambiente de apoio às conversas sobre os serviços públicos prestados. $O$ ambiente web está sendo desenvolvido em PHP e utiliza uma base de dados MySQL. Nele é disponibilizada uma visão geral da organização (seus objetivos e serviços prestados), a partir da qual os envolvidos têm visibilidade sobre o funcionamento dessa organização e podem manifestar-se sobre a prestação de serviços da mesma (Figura 4).

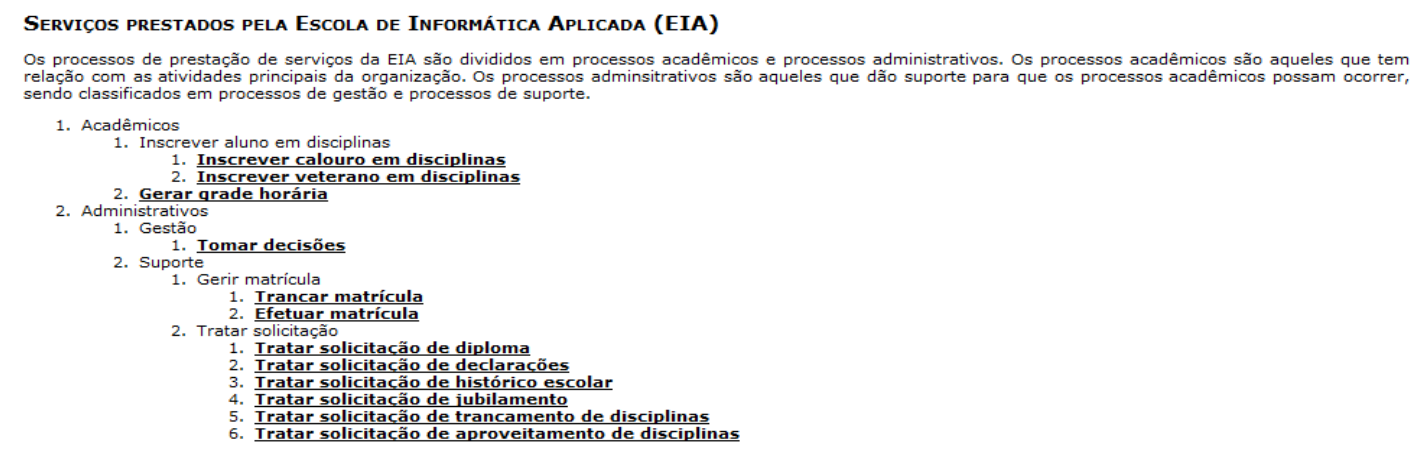

Figura 4. Visão geral da organização

A partir dessa visão geral, é possível acessar o processo detalhado de todos os serviços prestados pela organização. O detalhamento do serviço (Figura 5) é apresentado através de um modelo que descreve o seu funcionamento através dos principais elementos de processo, além de disponibilizar uma descrição para cada uma das atividades necessárias à execução desse processo. Esse detalhamento é útil para um maior entendimento por parte de quem precisa utilizar esses serviços, além de servir como centralizador das manifestações feitas pelos participantes.

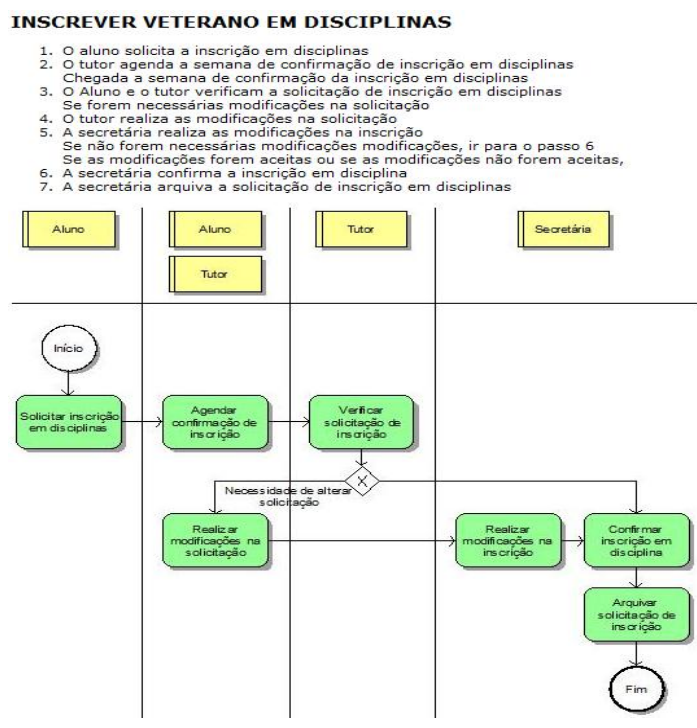

Figura 5. Detalhamento do serviço

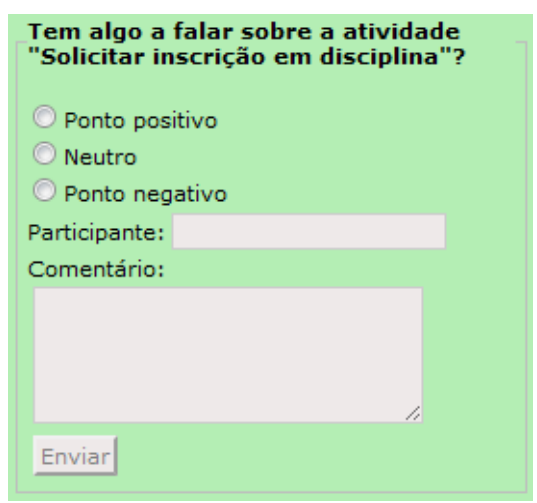

Figura 6. Manifestação ao elemento de processo "Atividade" 
Os envolvidos com os serviços prestados podem expor, de maneira global ou situada, as suas experiências, opiniões, dúvidas, problemas, sugestões, etc. sobre o processo apresentado. Os participantes devem apontar o elemento do processo para o qual a manifestação é feita e indicar se o relato é um ponto positivo, apenas uma informação ou um ponto negativo do processo (Figura 6). Essas ações possibilitam a associação entre as manifestações e os elementos do processo, auxiliam na organização das manifestações e facilitam a identificação de informações que podem ser relevantes para a melhoria do serviço.

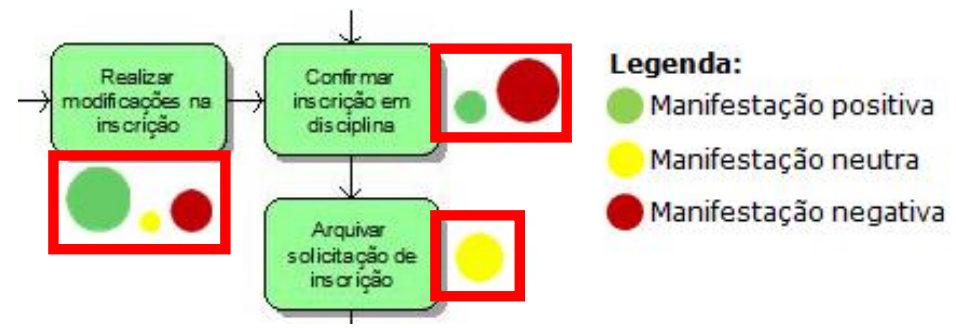

Figura 7. Visualização de manifestações aos elementos do processo

A partir do momento que as manifestações sobre o processo são classificadas, é possível gerar visualizações de informações que ajudam na percepção dos participantes (Figura 7). As visualizações são geradas para cada um dos elementos de modelos de processos. Elas ajudam na explicitação da existência de manifestações sobre o serviço e no entendimento dos assuntos abordados na discussão. Além disso, o conhecimento obtido a partir das manifestações pode ser invisível aos gestores do processo e, ao tornálo explícito, permite a realização de análises para identificação de melhorias no serviço público em pauta.

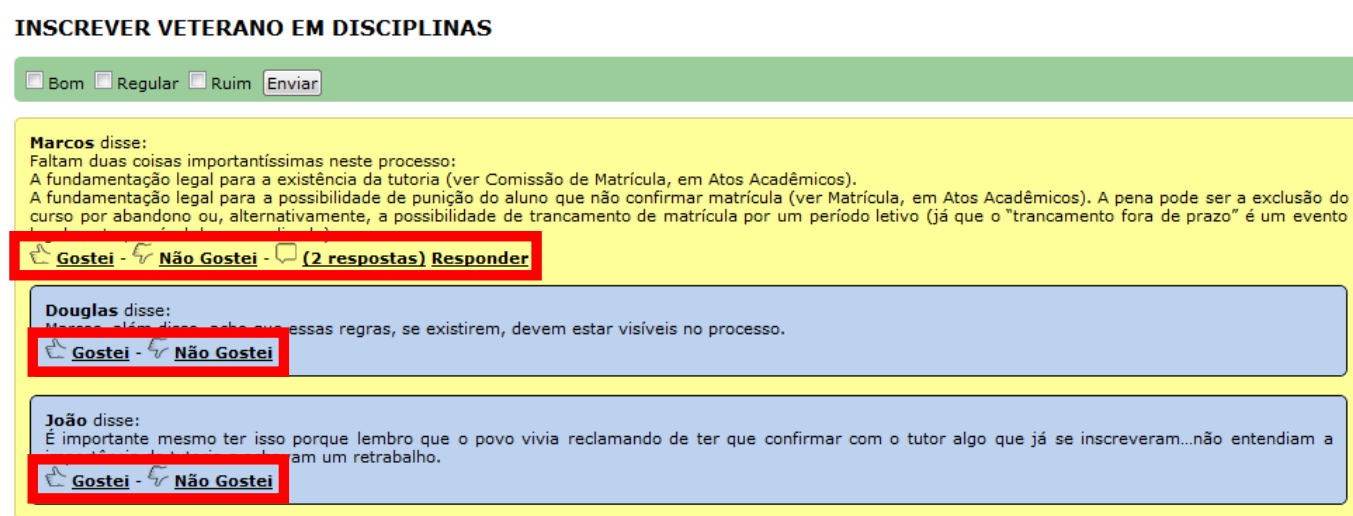

Figura 8. Mecanismos de interação

Caso queira conhecer detalhes das manifestações, os participantes podem visualizar as manifestações feitas em determinado ponto do processo. $\mathrm{O}$ ambiente apresenta as manifestações e fornece mecanismos que possibilitam a interação com as mesmas (Figura 8). Essa possibilidade de interação propicia o estabelecimento de diálogo entre os participantes, aumentando o conhecimento sobre o serviço e promovendo discussões. Ao conhecerem detalhes do serviço, os participantes podem relatar novas informações sobre o processo, problemas nas informações fornecidas previamente e sugestões para solucionar as dificuldades e inconsistências encontradas. As manifestações feitas podem ser utilizadas por outros participantes na argumentação 
de uma nova manifestação ou para complementar as informações fornecidas anteriormente.

Além de propiciar uma maior interação entre os cidadãos, as manifestações auxiliam a organização durante a análise do processo. $O$ gestor do processo pode recuperar as manifestações feitas, de acordo com determinados critérios, identificando e classificando os trechos do texto que indiquem a necessidade de alterações no processo.

\section{Conclusão}

O presente artigo apresenta uma proposta para estimular a aproximação entre Governo e Sociedade através de conversas sobre os processos de prestação de serviços públicos. É proposta a especificação de um ambiente onde conversas entre os envolvidos com os serviços prestados são feitas com base nos processos que descrevem o seu funcionamento. A disponibilização do processo mantém a conversa focada e dá visibilidade às informações de funcionamento dos serviços, que por muitas vezes são desconhecidas à Sociedade.

Os principais benefícios obtidos com a solução proposta são um maior envolvimento e participação da Sociedade nos serviços prestados pelo Governo; a possibilidade de interação entre os Cidadãos nas discussões sobre os serviços públicos; além da identificação de melhorias nos serviços prestados, que podem estar invisíveis aos gestores do mesmo, a partir das manifestações feitas pela Sociedade.

Como trabalhos futuros, será finalizado o desenvolvimento do ambiente de apoio às conversas sobre os serviços públicos prestados, além da realização de estudos utilizando o ambiente desenvolvido, no contexto da UNIRIO. Os estudos seguirão a abordagem adotada no projeto Ágora [ÁGORA, 2010][DIIRR et al, 2009][ENGIEL et al, 2010]. Serão disponibilizados para a comunidade os serviços prestados pela UNIRIO, de modo que os participantes conheçam e interajam com os serviços e entre si. Almeja-se com essa interação a obtenção de insumos que possibilitem a construção de visualizações das informações geradas [TAVARES et al, 2010], além da avaliação da utilização de conversas sobre processos públicos como mecanismo facilitador de uma maior interação entre Organização e seus integrantes.

\section{Agradecimentos}

Este trabalho está inserido no contexto do projeto Democracia, Transparência e Administração Pública - Reflexões e Implementações para o apoio a Sistemas de Governo através de TICs (http://www.uniriotec.br/ agora), financiado pela Coordenação de Aperfeiçoamento de Pessoal de Nível Superior (CAPES) e no contexto do Programa INC\&T - Projeto: Instituto Brasileiro de Pesquisa em Ciência da Web CNPq 557.128/2009-9 e FAPERJ E-26/170028/2008.

\section{Referências}

ADAMNS, D. (1979) “O guia do mochileiro das galáxias”, Sextante.

ÁGORA (2010) Projeto de pesquisa, http://www.uniriotec.br/ agora.

ALLEN, C. (2004) "Tracing the Evolution of Social Software", http://www.lifewithalacrity.com/2004/10/tracing_the_evo.html 
ARNSTEIN, S. (1969) “A ladder of citizen participation", Journal of the American Institute of Planners, v. 35, n.4, p. 216-224.

BRASIL. (2008) "Carta de Serviços ao Cidadão", http://www.governoeletronico.gov.br/anexos/apresentacao-carta-de-servicos-aocidadao

BRASIL (2010), http://www.brasil.gov.br

BRYANT, T. (2006) "Social Software in Academia", Educase Quarterly, n. 6, pp. 6164.

BUSINESS DICTIONARY (2010), http://www.businessdictionary.com/definition/ process.html

CARVALHO, E. (2009) "Engenharia de processos de negócios e a Engenharia de requisitos: Análise e comparações de abordagens e métodos de elicitação de requisitos de sistema orientada por processos de negócio", Dissertação de Mestrado UFRJ/COPPE/Programa de Engenharia de Produção.

CGI.BR (2010) “TIC Governo Eletrônico”, http://www.cetic.br/tic/egov/2010/index.htm

DIIRR, B.; ARAUJO, R. CAPPELLI, C. (2009) "An approach for defining Digital Democracy support based on ICT", In Proceedings of the 2009 13th International Conference on Computer Supported Cooperative Work in Design (CSCWD'09), pp. 203-208, Santiago.

DUTTA, S.; MIA, I. (2010) “The global information technology report 2009-2010 ICT for sustainability", In World Economic Forum.

ENGIEL, P.; ARAUJO, R. CAPPELLI, C. (2010) "Habilitando processos de prestação de serviços à participação e à Democracia", In Proceedings of IADIS Ibero Americana wwwInternet2010 (IADIS'10), Algarve.

FEMERS, S.; WIEDEMANN, P. (1993) "Public participation in waste management decision making: Analysis and management of conflicts", Journal of Hazardous Materials, v. 33, pp. 355-368.

GOMES, W. (2004) Transformações da política na era da comunicação de massa, Paulus.

GONÇALVES, J. (2010) "Story Mining: Elicitação de processos de negócio a partir de Group Storytelling e técnicas de mineração de texto", Dissertação de Mestrado UNIRIO/PPGI.

GONÇALVES, R.; PESSOA, M.; SPINOLA, M.; PRADO, J. (2005) “A importância de representar pessoas na modelagem de processos de negócio: Uma aplicação em reengenharia”, Encontro Nacional de Engenharia de Produção (ENEGEP).

HAGUE, B.N (1999) "Digital Democracy: Discourse and Decision Making in the Information Age", Routledge, 1st edition.

HWANG, J.; MANANDHAR, S. (2009) "Cost-benefit analysis of OPEN system: A case study for Kathmandu Metropolitan City", In Proceedings of the 4th International Conference on Computer Sciences and Convergence Information Technology (ICCIT'09), pp. 1425-1430, Seoul. 
MAIA, R. (2001) "Democracia e a internet como esfera pública virtual: aproximando as condições do discurso e da deliberação", Universidade de Brasília.

MACINTOSH, A. (2004) "Characterizing e-participation in policy-making", In Proceedings of the 37th Hawaii International Conference on System Sciences (HICSS), Hawaii.

MANY EYES (2010). Percentual de brasileiros em relação às redes sociais, http://www958.ibm.com/me

MIAH, S.; GAMMACK, J.; GREENFIELD, G. "An infrastructure for implementing eparticipation services in developing countries", In: Proceedings of the 3rd IEEE International Conference on Digital Ecosystems and Technologies (DEST'09), pp. 407-411, Istambul, 2009.

MINISTERIO DA CULTURA (2009). Consulta pública para reforma da Lei Rouanet, http://blogs.cultura.gov.br/blogdarouanet/

MINISTÉRIO DA JUSTIÇA (2010). Consulta pública do Marco Civil da internet, http://culturadigital.br/marcocivil/

MINISTÉRIO DA SAÚDE (2010). Consulta pública do Sistema Único de Saúde, http://200.214.130.94/consultapublica/index.php

OATES, S. (2008) Introduction to media and politics, Sage Publications.

OECD. (2001) "Citizens as partners", Handbook on information, consultation and public participation in policy-making.

OLIVEIRA, A. C.; ARAUJO, R.; BORGES, M (2007). "Telling Stories about System Use: Capturing Collective Tacit Knowledge for System Maintenance", International Conference on Software Engineering and Knowledge Engineering (SEKE), pp. 337342.

PORTAL DA PREFEITURA DO RIO (2010), http://www.rio.rj.gov.br.

REUVER, M.; STEIN, S.; HAMPE, F.; BOUWMAN, H. (2010) "Towards a service platform and business model for mobile participation", In Proceedings of the 9th International Conference on Mobile Business (ICMB'10), pp. 305-311, Atenas.

SHIRKY, C. (2008) "Here comes everybody: The power of organizing without organization", The Penguin Press.

SILVA, S.P. (2005) "Graus de participação democrática no uso da Internet pelos governos das capitais brasileiras”, Opinião Pública, vol. XI, no 2, pp. 450-468.

TAVARES, R.; PIMENTEL, M.; ARAUJO, R.; CAPPELLI, C. (2010) "Percepção de Tendências em Discussões Democráticas", In Proceedings of the 7th Simpósio Brasileiro de Sistemas Colaborativos (SBSC'10), p. 79-86, Belo Horizonte.

VAN DIJK, J.; HACKER, K. (2003) "The digital divide as a complex and dynamic phenomenon". The Information Society, v. 19, no 4, pp. 315-326.

W3C. (2009) "Melhorando o acesso ao governo com o melhor uso da web", http://www.w3c.br/divulgacao/pdf/gov-web.pdf 